\title{
Split-inducing indels in phylogenomic analysis
}

\author{
Alexander Donath ${ }^{1^{*}}$ (1) and Peter F. Stadler $2,3,4,5,6,7,8$ (B)
}

\begin{abstract}
Background: Most phylogenetic studies using molecular data treat gaps in multiple sequence alignments as missing data or even completely exclude alignment columns that contain gaps.

Results: Here we show that gap patterns in large-scale, genome-wide alignments are themselves phylogenetically informative and can be used to infer reliable phylogenies provided the gap data are properly filtered to reduce noise introduced by the alignment method. We introduce here the notion of split-inducing indels (splids) that define an approximate bipartition of the taxon set. We show both in simulated data and in case studies on real-life data that splids can be efficiently extracted from phylogenomic data sets.
\end{abstract}

Conclusions: Suitably processed gap patterns extracted from genome-wide alignment provide a surprisingly clear phylogenetic signal and an allow the inference of accurate phylogenetic trees.

Keywords: In/del, Splits, Genome-wide multiple sequence alignments, Phylogenomics

\section{Background}

Gaps in multiple sequence alignments are usually seen as a nuisance in molecular phylogenetics. In most studies, gaps are treated as missing data or alignment columns with gaps are even removed completely. Indeed, stochastic models of sequence evolution that deal explicitly with insertions and deletions (indels) have been investigated only recently $[1,2]$. Detailed evaluation shows an overall improvement of phylogenetic reconstructions when indels are modelled explicitly [3-5]. For instance, the inclusion of insertion and deletion (indel) characters proved useful in the analysis of the phylogeny of the Arctoidea (Mammalia: Carnivora) [6], neognathous birds [7], or fungal families [8]. Nevertheless, there is a negative effect of an increasing density of gap characters in multiple sequence alignments [5]. Furthermore, recent studies have indicated that biases may be introduced when indels are included without precautions in Bayesian and Maximum Likelihood phylogenies $[9,10]$.

\footnotetext{
*Correspondence: a.donath@leibniz-zfmk.de

${ }^{1}$ Center for Molecular Biodiversity Research (zmb), Zoological Research Museum Alexander Koenig (ZFMK), Adenauerallee 160, 53113 Bonn, Germany

Full list of author information is available at the end of the article
}

Between these few recent rigorous approaches to include gaps and the dismissal of gaps as missing data, indels have been incorporated in several ways into sequence-based phylogenetic analyses. The simplest one is the coding of gaps as fifth character state. Other authors have suggested the replacement of the gapped regions by a binary matrix that codes presence and/or absence of the respective indel [11]. This binary matrix is then added to the "ungapped" sequence data and employed in tree inference. An extension of this simple indel coding (SIC) approach maximizes the amount of phylogenetic information in a parsimonious way by incorporating all indels [12].

Gaps in alignments are, of course, not features identifiable from the individual sequences. Instead, they appear as derived patterns inferred from sequence comparison only. Nevertheless, they convey a surprising amount of phylogenetic information. Shared multi-residue deletions, for instance, have been used to support hypothesis derived from molecular data in single gene analyses, see e.g. [13]. Multi-residue gaps in nucleotide as well as protein sequences have been reported as useful indicators of monophyletic groups [14]. Single-residue gaps, on the other hand, occur more frequently than multi-residue 


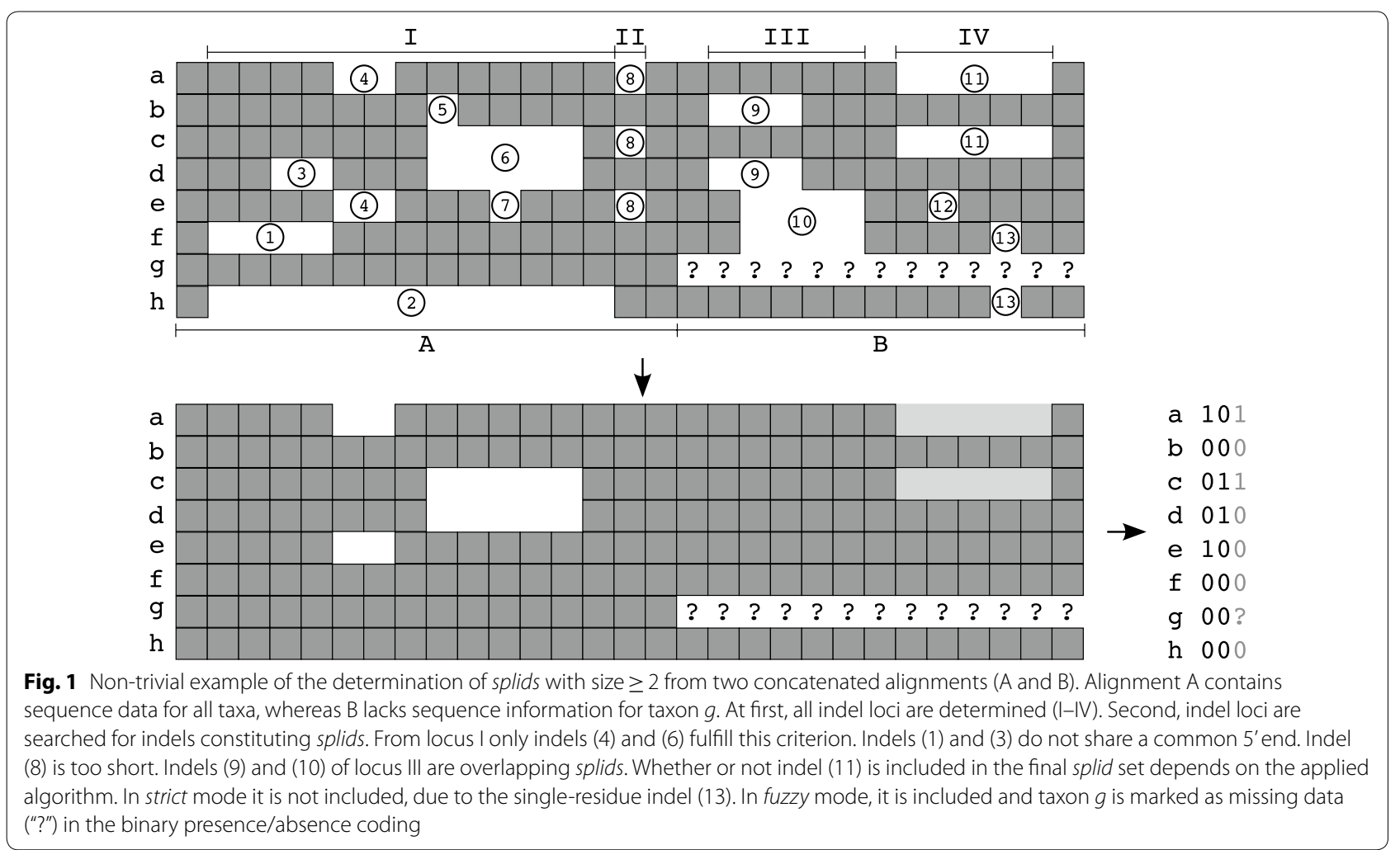

gaps and show a higher amount of homoplasy, e.g. [15]. The same authors suggest that single-residue gaps should not be removed a priori from a data set based on a large taxon sampling, since they can still contain a phylogenetic signal. Ashkenazy et al. [16] proposed to quantify the reliability of indel characters by measuring the frequency with which they appear in alternative multiple sequence alignments. They show that weighting or filtering indels by reliability in general improves the accuracy of phylogenetic reconstruction.

The few studies of the phylogenetic information content of gap patterns were mostly conducted on limited sets of protein data. Gap patterns are, however, very different between coding and non-coding regions [17]. With the advent of high-throughput sequencing (nearly) complete genomes are becoming available at an increasing pace, from which large-scale genome-wide alignments can be constructed [18, 19]. Phylogenomics capitalizes on these developments and provides a wide diversity of phylogenetic information [20]. We utilize these developments here to address the value of gap patterns from a phylogenomic perspective. Since we aim at using pre-computed genome-wide alignments it is not feasible to evaluate individual gaps by their stability with regard to different alignment methods as proposed in [16]. On the other hand, the size of genome-wide data sets allows us to devise stringent filtering criteria to reduce noise and alignment-specific biases. To this end we focus on the sub-class of indels that define a "reasonably obvious" binary split among the sequences. As gaps are not part of the sequence itself but the result of an alignment algorithm, however, we need to systematically investigate the impact of the alignment method on the phylogenetic information of the gap patterns.

\section{Theory: inference of split-inducing indels}

The encoding of characters from gap patterns is not entirely trivial as soon as indels rather than individual gap characters are to be assessed.

We formally define an indel to be a contiguous stretch of gap characters in one or more rows of the alignment. Each indel therefore has a well-defined start and stop column. Its size is defined as the number of consecutive gap characters. Two indels overlap if there is an alignment column that is common to both of them [see, e.g., indel (1) and (3) in Fig. 1]. An indel locus consists of indels that overlap, i.e., a contiguous sequence of alignment columns such that two adjacent columns share at least one indel. By definition, the indels in two indel loci are independent of each other and thus can be treated separately. 
We call an indel a split-inducing indel (splid) if it defines an approximate bipartition of the taxon set according to the following rules:

1. Only indels that are present in at least two sequences and have a user-defined minimum size are taken into account. By default, all indels of size at least two are considered. Thus, indels (1), (2), (3), (5), (7), (8), (12), and (13) in Fig. 1 can be ignored.

2. A splid cannot overlap another indel that satiesfies the first condition. Thus, indels (9) and (10) are excluded.

Splids are coded as binary characters marking their presence/absence pattern in the respective taxon. Missing sequence data in the alignment column of a splid is coded as "missing data" ("?"). We optionally filter out splids that overlap a single-residue indel occurring in at least two taxa [such as indel (13)]. Applying this "strict mode" removes indel (11), while it is retained in "fuzzy mode". These alternative treatments of single-position gaps is motivated by the observation that they occur more randomly than multi-residue gaps, while still containing some phylogenetic information [15]. Thus, including these splids could increase the number of available characters, although this increases the possibility of conflicting signal.

\section{Methods}

\section{Implementation}

The algorithm for the conversion of alignments to a binary character matrix is implemented in the $\mathrm{C}++$ program gappy. It reads multiple sequence alignments in MAF and FASTA format. The user can select a minimum and maximum indel size for determining splids. By default, the output is a FASTA file, containing the binary coded splid presence/absence information, and a summary statistic with details about the identified splids. Output is also available in PHYLIP and NEXUS format.

\section{Data sets \\ Simulated data}

Indel rates and indel-size distributions are usually estimated based on pairwise alignments (e.g., human-mouse, primates, rodents [21-24]) but differ quite considerably. For example, estimates for the ratio of substitution rates to indel rates between mouse and human are ranging from 8 [24] to 14 [22, 23]. It seems to be a good approximation to apply an indel rate in vertebrates at least as high as between human and mouse, however. Estimates suggest that the frequency of deletions is somewhat higher than the insertion frequency $[21,25,26]$, with a ratio of deletion rate $\lambda_{d}$ to insertion rate $\lambda_{i}$ ranging from 1.3 to 4 .
We therefore created three different data sets using the F81 model [27], two indel-size distributions and different indel rates, each consisting of 100 alignments with a length of 100,000 bp (see Additional file 1: Figure S1). The first two data sets use a geometric distribution with similar insertion and deletion rates $\left(\lambda_{i 1}=\lambda_{i 2}=0.03106\right.$ and $\left.\lambda_{d 1}=\lambda_{d 2}=0.04037\right)$ but different probability values ( $q_{1}=0.7$ and $q_{2}=0.55$, respectively). The third data set follows a Lavalette distribution $(a=1.5, M=120$, $\lambda_{i 3}=0.02899$, and $\lambda_{d 3}=0.03768$ ), which has been suggested as being an appropriate approximation of the indel length distribution in real-life data sets $[28,29]$. All data sets were simulated using INDELible V1.03 [29]. The guide tree and background base frequencies were taken from the phastCons17way phastCons tree model file [30] obtained from $\mathrm{UCSC}^{1}$ and rescaled to have a maximum root-to-tip distance of 2 .

\section{ENCODE data}

In order to address the problem of how our method behaves under real-life data and genome-scale alignment lengths we created two data sets from the ENCODE [31] project data, based on the December 2007 Multi-Species Sequence Analysis sequence freeze available from UCSC. $^{2}$ The ENCODE data contains sequences of 35 vertebrates orthologous to a representative $1 \%$ of the human genome divided among 44 regions. The sequences were aligned with TBA/Multiz [18], a toolkit that has been widely used for whole-genome alignments in large-scale comparative genomics studies [31, 32]. TBA/Multiz produces a set of local alignments ("blocks") that are stitched together relative to a reference sequence to represent the evolutionary operations, in particular insertions and deletions, that separate the included sequences. The program requires a predefined guide tree that describes the relationship of the species to be aligned. In case of the ENCODE data set this tree is largely based on taxonomic information.

A genome-wide alignment is the result of an extensive similarity search between at least two species. Due to evolutionary changes in genome organization, such as inversions and duplications, two genomes are virtually never completely co-linear, resulting in a decomposition of alignments into syntenic blocks. Practical procedures such as TBA/Multiz also use other features, such as large insertions, missing data in individual species, or low complexity regions, as additional breakpoints, so that relative small alignment blocks are produced. Not all of

\footnotetext{
${ }^{1}$ http://hgdownload.cse.ucsc.edu.

${ }^{2}$ http://hgdownload.cse.ucsc.edu/goldenPath/hg18/encode/MSA/DEC$2007 /$.
} 
these blocks contain sequence information from all taxa, both due to missing data in the sequence assemblies and because highly diverged regions of some taxa cannot be reliably recognized as homologous.

The first data set contains only those alignments with sequence information for all 36 organisms. Alignment blocks of two ENCODE regions fulfilled this criteria: ENm001 (498 alignment blocks) and ENm013 (67 alignment blocks). To investigate how the method behaves under a considerable amount of missing data, as it is usually the case for genome wide alignments, we created a second data set, based on all ENCODE alignment regions with sequence information for at least three species.

\section{Re-alignment without predefined guide trees}

The use of a predefined guide tree for the genome alignments could conceivably create a bias in indel positioning. We therefore checked whether such a bias exists and how other commonly used alignment programs perform. To this end we removed all gaps from the ENCODE alignment blocks. The genome-wide alignments thus are used only as a convenient means of extracting orthologous regions.

We applied a similar procedure to the 'true' alignments of the simulated data set. To mimic the properties of the ENCODE alignments, we first split all simulated alignments in blocks with an average size of $140 \mathrm{bp}$. After removing all gaps, each block was then re-aligned with a variety of commonly used multiple sequence alignment programs using default settings: ClustalW version 2.0.12 [33], Muscle version 3.7 [34], T-Coffee version 8.97 [35], Prank version 100802 [36], Dialign-TX version 1.0.2 [37], and Mafft version 6.833b [38]. Mafft was run in three different strategies: default mode, L-INS-i, and G-INS-i mode. Dialign-TX differs from all other methods as it creates alignments from local pairwise sequence similarities without the use of explicit gap penalties.

Approximately 2\% of the ENCODE regions contain coding exons while the majority covers non-coding sequences, such as introns, UTRs, and intergenic regions. It has been pointed out that, while performing fairly good on these sequences, TBA/Multiz's results on regions containing non-coding RNAs is not optimal [39]. We therefore additionally tested ProbConsRNA version 1.1 [40], an experimental version of PROBCONS for nucleotide data with parameters estimated from BRAliBASE II via unsupervised training [41].

Following realignment, gaps introduced at the $5^{\prime}$ and $3^{\prime}$ ends of the sequence blocks were considered as artifacts and hence coded as missing data (see also [11]). As individual alignment blocks typically contain sequence information for only a subset of the input taxa, sequences of such missing taxa were also explicitly coded as missing data. Alignment blocks with sequence information for two or more taxa and containing at least one gap character were then concatenated using a custom Perl script (available with the source code of gappy). Note that by construction the delimiting columns of each alignment block do not contain gap characters; concatenation therefore does not affect the gap patterns. From these concatenated alignments we extracted all splids $\geq 2 \mathrm{bp}$ using gappy in strict mode.

\section{Phylogenetic reconstruction and analysis Model selection and tree reconstruction}

Binary model selection was performed using PartitionFinder version 2.1.1 [42] and comparing the BIC scores. Phylogenetic trees were calculated with RAxML version 8.2.11 [43], executing 100 rapid bootstrap inferences and thereafter a thorough ML search. Bootstrap support values were drawn on the best-scoring tree.

\section{Tree comparison}

Two phylogenetic $n$-taxa trees can be compared using a variety of different distance measures. The most sensitive one is the unweighted Robinson-Foulds (RF) distance $\left(d_{R F}\right)$ [44], defined as the sum of the number of splits present in exactly one of the two trees. The normalized RF distance $\left(d_{R F}^{\prime}\right)$ is then computed by dividing $d_{R F}$ by the maximal possible distance between the two trees, i.e., $d_{R F}^{\prime}=d_{R F} /(2 n-6)$. The RF measure does not emphasize local similarity, so that trees differing by the placement of a single taxon may have a large RF distance [45]. We therefore also calculated the quartet distance $\left(d_{Q}\right)$ [46], defined as the number of quartets that are subtrees of one but not the other input tree, for comparison. The normalized quartet distance, $d_{Q}^{\prime}=d_{Q} /\left(\begin{array}{l}n \\ 4\end{array}\right)$, serves as

a convenient distance measure between large phylogenetic trees. We use here Phylonet version 3.6.1 [47] and tqDist version 1.0.0 [48] to compare the obtained trees with the respective UCSC guide trees.

\section{Results}

\section{Simulated alignments}

In order to test the phylogenetic signal provided by splids we first used simulated sequence data generated with INDELible along a known reference tree. Alignments were computed using nine different methods. Partitionfinder identified the GAMMA model of rate heterogeneity including ascertainment bias correction as the most suitable model for all splid alignments. In total 3000 trees were calculated from these alignments and the simulated INDELible reference alignments. On these artificial data set we observe nearly correct trees 
Table 1 Overview of the total number of sites of all alignments per alignment method and the number of derived splids with length $\geq \mathbf{2}$ bp for the ENCODE data set containing only alignments with sequence information for all taxa

\begin{tabular}{lcc}
\hline Program & Number of sites & $\begin{array}{c}\text { Number } \\
\text { of splids }\end{array}$ \\
\hline ClustalW & 79,006 & 793 \\
Dialign-TX & 96,990 & 2163 \\
Mafft & 84,105 & 1021 \\
Mafft L-INS-i & 83,578 & 1245 \\
Mafft G-INS-i & 83,123 & 1279 \\
Muscle & 84,577 & 1378 \\
ProbConsRNA & 86,277 & 1927 \\
Prank & 96,622 & 2047 \\
T-Coffee & 84,835 & 1831 \\
TBA/Multiz & 90,726 & 2032 \\
\hline
\end{tabular}

derived from splids (see Additional file 1: Figure S1). On these benign data, the choice of the alignment methods has little effect on the quality of the estimated phylogenies. No RF distances between reconstructed phylogeny and reference tree larger than 4 were observed. This corresponds to a maximum of two splits that are not present in the reference tree. Indeed $84.07 \%$ of the trees were identical to the reference tree, and another 15.17\% showed an RF distance of 2. Quartet distances draw a similar picture but allow a better differentiation between the results of the respective methods. The overwhelming majority of all trees (97.4\%) from all alignment methods have a $d_{Q}^{\prime} \leq 0.001221$. The tree most dissimilar to the guide tree $\left(d_{Q}^{\prime}=0.016801\right)$ was calculated based on one of the ClustalW alignments. The alignment program that performed best in terms of similarity to the reference tree was Mafft L-INS-i with an average of $d_{Q}^{\prime}=0.000227$.

\section{ENCODE genomes}

Data set with sequence information for all taxa. Depending on the alignment method, the concatenated alignments of the ENCODE data differed quite considerably in length and hence in the total number of gaps. For the small ENCODE data set, ClustalW produced the shortest and Dialign-TX the longest alignment (Table 1). In general, the number of splids increased with the number of alignment sites. For the three Mafft algorithms, however, the number of splids decreases with increasing alignment length. In particular, Mafft default and Mafft L-INS-i seem to introduce more single-residue gaps or conflicting splits than Mafft G-INS-i.

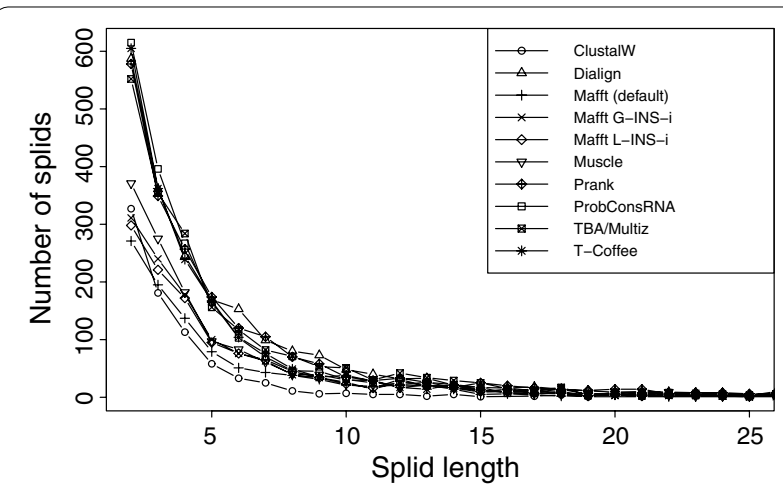

Fig. 2 Number of splids with a length of $\geq 2$ bp that have been extracted from the alignments of the ENCODE data set containing sequence information for all taxa

Dialign-TX, T-Coffee, Prank, and ProbConSRNA yield a splid length distribution similar to TBA/ Multiz (Fig. 2). In comparison, Muscle, clustalw, and all three Mafft algorithms found considerably fewer shorter splids. There is, however, no systematic dependence on design features of the alignment methods such as global versus local alignments or progressive versus consistency based methods.

While the splid-based phylogenies are nearly perfect on simulated data, we observed larger deviations that depend at least in part on the alignment methods when applying our approach to real-life data. On the other hand, in real data sets we do not have an absolute ground truth to compare to. Thus, we discuss in following both the quality of the reconstructed phylogenies and the position of interesting taxa in some detail [see Table 2 and machine-readable data online (see Availability)].

The monophyly of Afrotheria and the positioning of tenrec basal to elephant and rock hyrax $[49,50]$ was always recovered. Only splid data derived from the Mafft default alignments placed tenrec basal to armadillo. The position of the placental root is still, at least to some extent, a matter of debate [51-54]. However, results based on the Mafft default alignments and most other alignment programs correctly positioned Afrotheria outside of Boreoeutheria [55]. Only splid data obtained from the Muscle, ProbConsRNA, and $\mathrm{T}$-Coffee alignments placed Afrotheria as sister group to Laurasiatheria (ProbConsRNA and T-Coffee) or inside Euarchontoglires (Muscle). The original TBA/ Multiz alignments did not contain enough supporting splids to position Afrotheria outside of Boreoeutheria, however.

Three hypotheses concerning the positioning of Xenarthra are discussed in the literature: (1) basal-Afrotheria ((Boreoeutheria, Xenarthra); Exafroplacentalia), e.g. 


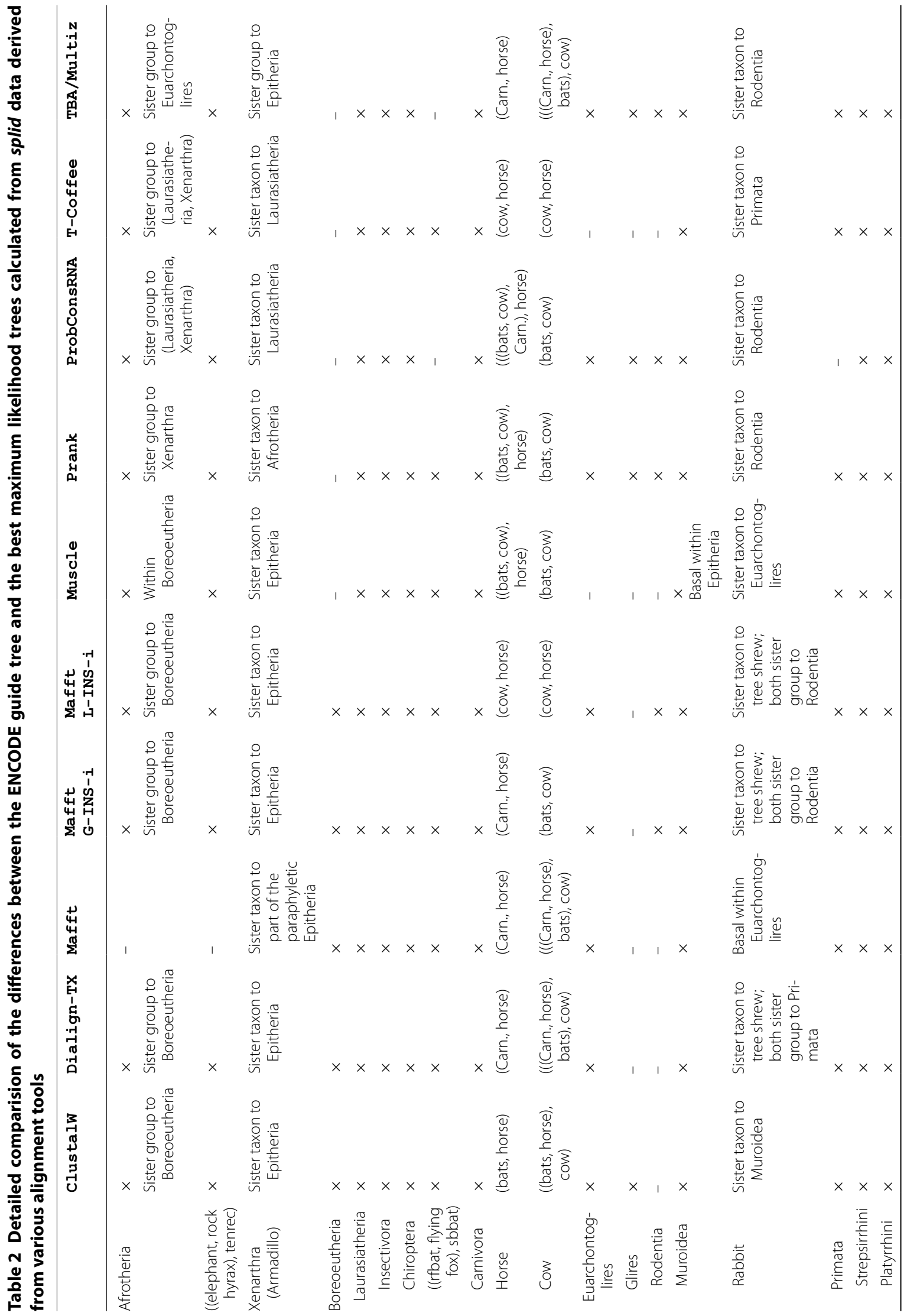




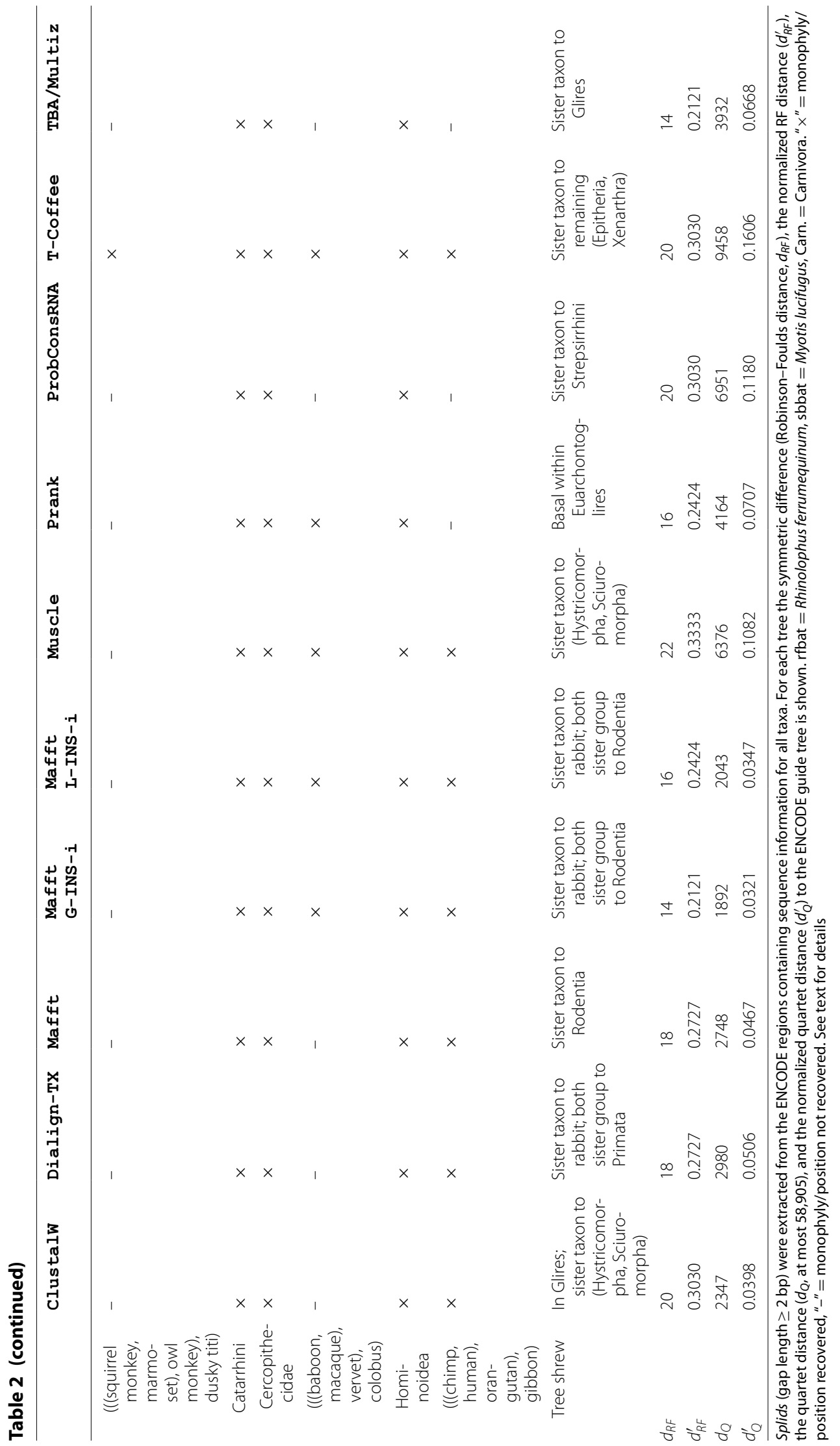


[51, 54], (2) basal-Xenarthra ((Boreoeutheria, Afrotheria); Epitheria), e.g. [56], and (3) basal-Boreoeutheria ((Afrotheria, Xenarthra); Atlantogenata), e.g. [57]. Splid data mostly supports the basal-Xenarthra hypothesis. Prank positioned armadillo basal to Afrotheria, whereas ProbConsRNA and T-Coffee placed armadillo basal to Laurasiatheria and therefore inside Boreoeutheria. Interestingly, none of the calculated trees supports the ENCODE guide tree that follows the basal-Afrotheria hypothesis.

Monophyly of Laurasiatheria was recovered in all cases. Monophyly was also recovered for its major orders Insectivora (Eulipotyphla), Chiroptera, and Carnivora. There was no clear result from splid data about the relationship within Laurasiatheria, which resembles the conclusions obtained elsewhere [50,52, 55], although results from all alignment methods support Insectivora (Eulipotyphla) as the most basal clade within Laurasiatheria [52]. The evolutionary history of bats has long been a subject of discussion, with conflicting hypothesis depending on whether morphological or molecular data was used. Earlier studies either traditionally suggested the monophyly of the suborders Megachiroptera (megabats) and Microchiroptera (microbats), e.g. [58], while other studies placed megabats together with the rhinolophoid microbats (Yinpterochiroptera), with the remaining microbats forming the suborder Yangochiroptera, e.g. [59, 60]. Splid data derived from most of the alignment methods support this and place Rhinolophus ferrumequinum as sister taxon to Pteropus vampyrus, while Myotis lucifugus was found as sister taxon to both of them. Only ProbConSRNA follows the traditional view of a monophyly of megabats and microbats and is therefore similar to the results obtained from the TBA/Multiz alignments.

The monophyly of Euarchontoglires (Euarchonta and Glires) could not be recovered from splid data obtained from Muscle and T-Coffee, because of the wrongly positioned Muroidea (Muscle and T-Coffee) and the wrong position of tree shrew, guinea pig, and squirrel (T-Coffee). However, all other alignment methods clearly support the monophyly of the superorder Euarchontoglires.

Among all analyzed groups, Glires are the most problematic one. Incongruent results were reported in the literature in particular concerning the position of the tree shrew. While some studies place tree shrews as sister group to Glires, others recover them as sister group to Primata (see [61] for a recent summary). Splid data also do not yield an unambiguous conclusion. They often place the tree shrew within (ClustalW, Mafft G-INS-i, Mafft L-INS-i) or in a sister group relationship to Glires (TBA/Multiz). Only splid data derived from ProbConsRNA alignments places the tree shrew (but also monophyletic Glires) within Primata. Interestingly, splid data obtained from the alignments of Prank recovered the tree shrew as the most basal taxon in Euarchontoglires (with monophyletic Glires as sister group to all Primates). Splid data from Dialign-TX, Mafft default, Muscle, and T-Coffee alignments recover Glires as polyphyletic with varying positions of the tree shrew.

Almost all methods support the monophyly of Primates, as well as a monophyly of the respective sub- and parvorders. Only splid data derived from the ProbConsRNA alignments places Strepsirrhini together with the tree shrew as the most basal clade within Euarchontoglires.

As a quantitatively evaluation of the mammalian tree we consider the normalized RF and quartet distances to the ENCODE reference tree, which-although not undisputed - well reflects the state of the art in mammalian phylogeny. Overall, the tree calculated from splids derived from the Mafft G-INS-i alignments shows the highest similary to the ENCODE reference tree (Fig. 3). The tree based on splids derived from the T-Coffee alignments is most different from the ENCODE tree with respect to the more sensitive quartet distance. While the tree computed with Muscle has a higher normalized RF distance, its normalized quartet distance is much lower. However, when comparing the values of the two distances for the other methods it becomes apparent that their results are quite different and show no clear correlation. For example, while the RF distances of the ENCODE tree to the trees based on the splids derived from the Mafft L-INS-i and Prank alignments, respectively, are similar, the quartet distances differ by a factor larger than two. Interestingly, when comparing the much more sensitive quartet distances of the trees based on splids extracted from the alignments calculated with ClustalW, Dialign-TX, and all three Mafft algorithms, they indicate a higher similarity to the ENCODE reference tree than the tree based on splids extracted from the guide tree based TBA/Multiz alignments. The Probabilistic Alignment Kit Prank [36] has been developed with a focus on a phylogenetic consistent placement of insertions and deletions. However, trees calculated from splids derived from Prank alignments showed no superior similarity to the ENCODE reference tree, an observation that is in line with another study [62]. We note, finally, that misplaced taxa in all trees generally had low bootstrap support.

Data set with sequence information for at least three species. In the following, we focus on three alignment methods to analyze splid performance on the large ENCODE data set: Mafft G-INS-i was chosen because it performed best on the data set containing 


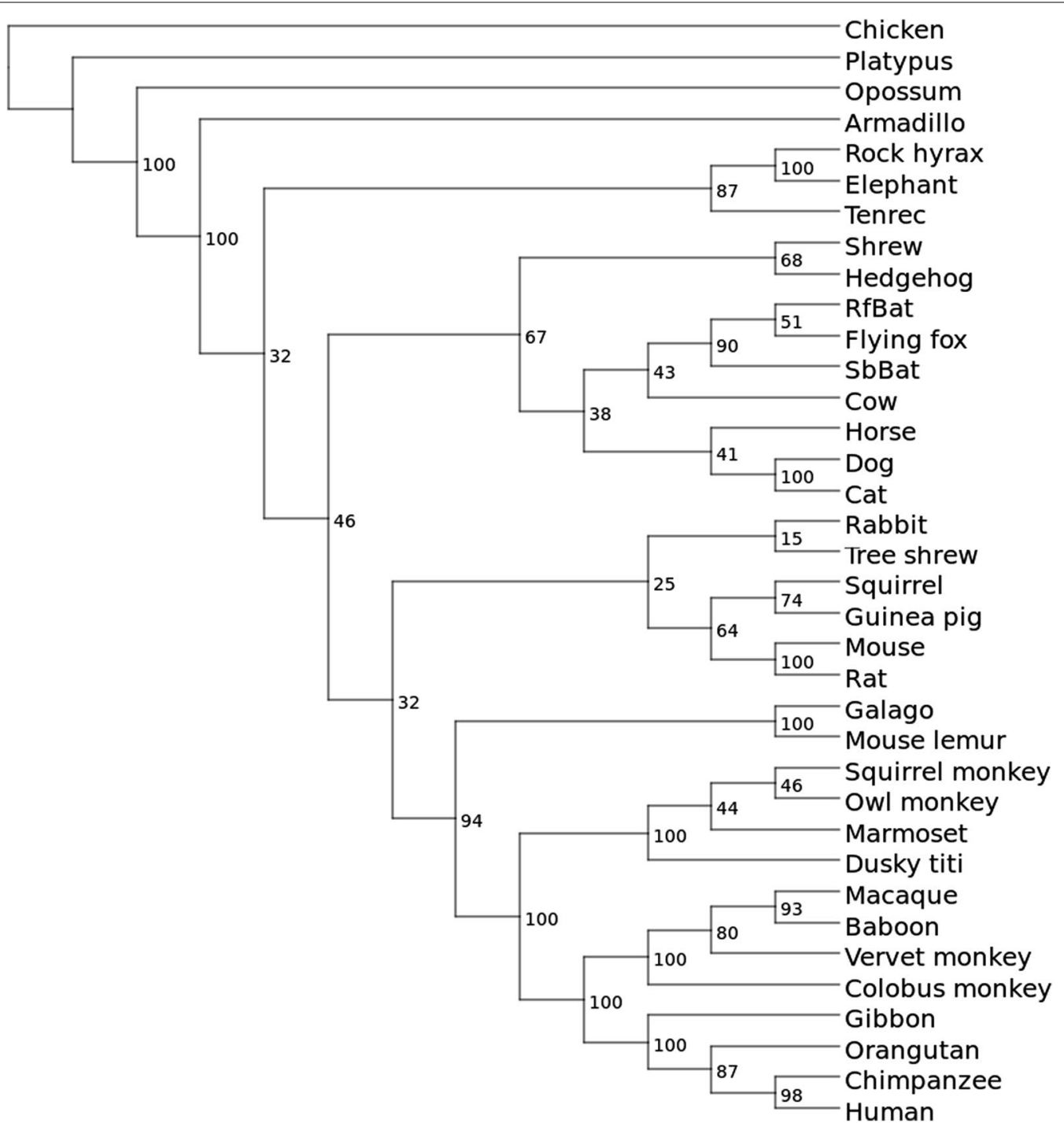

Fig. 3 Cladogram with bootstrap values obtained from 100 bootstrap trees calculated by RAxML using splid data and the Gamma model with ascertainment bias correction. Splids with gap lengths $\geq 2$ bp were extracted from the small ENCODE data set that has been re-aligned using Mafft G-INS-i

sequence information for all taxa. In order to analyze whether the increase in the size of the data set improves the performance, we also included T-Coffee, the method with the poorest performance on the small ENCODE data set (with respect to the quartet distance). In addition, we included in our analysis the splid set derived from the original TBA/Multiz alignments. We removed four invariant splid sites extracted from the TBA/Multiz alignments, because invariant sites are not allowed when ascertainment bias correction is used.

We observed an improvement in terms of tree similarity to the ENCODE guide tree for two of the three splid data sets derived from the large ENCODE data
Table 3 Results for the large ENCODE data set. Splids $\geq 2$ bp were coded and trees were calculated with RAxML using the Gamma model for binary data and ascertainment bias correction

\begin{tabular}{llll}
\hline & Mafft G-INS-i & T-Coffee & TBA/Multiz \\
\hline Number of sites & $36,132,992$ & $36,450,667$ & $37,689,662$ \\
Number of splids & 545,790 & 922,277 & 919,908 \\
$d_{R F}$ & 16 & 16 & 12 \\
$d_{R F}^{\prime}$ & 0.2424 & 0.2424 & 0.1818 \\
$d_{Q}^{\prime}$ & 5000 & 7494 & 3710 \\
$d_{Q}^{\prime}$ & 0.0849 & 0.1272 & 0.0630 \\
\hline
\end{tabular}


set (Table 3). For the T-Coffee alignments, both tree distance measures indicate higher similarity of the maximum likelihood (ML) tree to the ENCODE guide tree $\left(d_{R F}^{\prime}=0.2424\right.$ and $d_{Q}^{\prime}=0.1272$, respectively) than the tree calcuated from splid data derived from the small data set $\left(d_{R F}^{\prime}=0.3030\right.$ and $d_{Q}^{\prime}=0.1606$, respectively). However, Boreoeutheria were not found to be monophyletic anymore: Glires are placed as sister group to ((Afrotheria, Xenarthra), Laurasiatheria) and the remaining Euarchontoglires (Primata); although with low bootstrap support. The tree shrew is recovered in a sister taxon relationship to all of the former. On the other hand, monophyly of all other major groups (Laurasiatheria, Afrotheria) and groups therein (Chiroptera, Carnivora, Insectivora, Primata etc.) was correctly recovered.

$\mathrm{RF}$ and quartet distance of the ML tree calculated from splids derived from the original TBA/Multiz alignments also decreased $\left(d_{R F}^{\prime}=0.1818\right.$ and $d_{Q}^{\prime}=0.0630$, respectively) compared to the small ENCODE data set $\left(d_{R F}^{\prime}=0.2121\right.$ and $d_{Q}^{\prime}=0.0668$, respectively). Monophyletic Afrotheria are recovered as sister-group to monophyletic Boreoeutheria (Epitheria) with basal Xenarthra. Within Boreoeutheria, monophyly of all major groups were correctly recovered and order within groups largely follows the ENCODE guide tree with three notable exceptions. (1) The tree shrew is now recovered as sister taxon to (Epitheria, Xenarthra). (2) Insectivora (hedgehog and shrew) is not the basal group within Laurasiatheria anymore but is now sister group to Chiroptera, (3) while both are sister group to ((Carnivora, cow), horse).

Unexpectedly, the ML tree calculated from the Mafft G-INS-i alignments (Table 3) showed a higher distance to the ENCODE guide tree tree $\left(d_{R F}^{\prime}=0.2424\right.$ and $d_{Q}^{\prime}=0.0849$, respectively) and is thus more dissimilar than the tree calculated from splid data derived from the small data set $\left(d_{R F}^{\prime}=0.2121\right.$ and $d_{Q}^{\prime}=0.0321$, respectively). Here, Laurasiatheria were not recovered to be monophyletic. Instead, non-monophyletic Insectivora are recovered as basal to ((Afrotheria, Xenarthra), Euarchontoglires) and the remaining Laurasiatheria. Again, monophyly of all other major groups (Afrotheria, Euarchontoglires) and groups therein (Primata, Glires etc.) was correctly recovered and the tree shrew was placed as sister taxon to Glires.

We note, finally, that of all species included in the large ENCODE data set, tree shrew has by far the smallest sequence coverage (approximately $10 \%$ of the amount of human sequence in the alignments), which likely contributes to its unstable position.

\section{Discussion}

Indels are not features of individual sequences. Instead they are inferred by comparative analysis and, in practice, appear as gaps in multiple sequence alignments. In some alignment methods they are explicitly modelled and contribute to the score, e.g. by means of affine gap costs. In other approaches they are modelled only implicitly. It is not unexpected, therefore, that the number and position of gaps depends quite strongly on the alignment algorithm. The fact that the choice of the alignment algorithm has an impact on the reconstructed phylogenies is well documented in the literature, see e.g. [63-67]. Nevertheless, gap positions can be phylogenetically informative.

We have focused here on a subclass of indels, namely those which can be found in more than one sequence and therefore define a split in the taxon set. Our definition and inference of such split-inducing indels (splids) is based on two basic principles that are largely accepted in the literature. First, indels at the same position, i.e. sharing the same end points in two sequences, are likely homologous. Second, independent single-residue insertions and deletions tend to occur more frequently than multi-residue indels. Hence they are expected to contribute a more noisy signal and hence are disregarded in our analysis.

We have tested the information content of splids on three simulated and two real-life data sets and analyzed the capability of splids introduced by nine different alignment programs for phylogenetic inference by ML. For artificial data sets, which are generated from a known underlying phylogeny, we find that splid-based ML reconstruction leads to nearly perfect trees. On the reallife data sets, however, we observe larger discrepancies between different alignment methods.

The splid-based phylogenies clearly recovered most of the undisputed monophyletic groups. Although there are clear differences in the alignment methods, the approach is surprisingly robust across a wide variety of alignment techniques. We expected a large influence of the guide tree on the reconstructed phylogeny since guide trees are known to influence the indel pattern [68]. Nevertheless, we observed that this effect is small for indel-based phylogenies when only splids are considered. Overall, alignment methods that put more emphasis on modelling indels, in particular those that employ an affine gap cost model, perform superior to alignment algorithm that consider indels only implicitly. Furthermore, for very large data sets we can observe a decreasing influence of the alignment algorithm.

Similar to other phylogenetic approaches, taxon sampling has a major influence on branch positions in very divergent taxonomic orders. This can been seen for example in the Laurasiatheria, where a small group 
of more closely related taxa (e.g. bats or Carnivora) is embedded in a larger set of more distantly ones. While splid data always supports a monophyly of Chiroptera, their position within Laurasiatheria cannot be unambiguously determined.

Increasing sequence length, and therefore splid information, does not necessarily lead to better resolved trees. This effect is likely related to the observation that alignments computed for large data sets have relatively large error rates, especially when sequence coverage is low. This in turn introduces considerable conflicting signal in tree inference [69]. In the case of low but roughly equal amount of sequence data for all taxa, the choice of the alignment algorithm seems to have a higher effect within lower taxonomic orders, while groups resembling higher taxonomic orders are relatively stable and are mostly correct positioned. ${ }^{3}$

\section{Additional file}

Additional file 1: Figure S1. Normalized quartet distances for the simulated data sets.

\section{Authors' contributions}

Both authors designed the study and wrote the manuscript. AD implemented the gappy software and conducted the computational analysis. Both authors read and approved the final manuscript.

\section{Author details \\ ${ }^{1}$ Center for Molecular Biodiversity Research (zmb), Zoological Research Museum Alexander Koenig (ZFMK), Adenauerallee 160, 53113 Bonn, Germany. ${ }^{2}$ Bioinformatics Group, Department of Computer Science, Interdisciplinary Center for Bioinformatics, Universität Leipzig, Härtelstraße 16-18, 04107 Leip- zig, Germany. ${ }^{3}$ Competence Center for Scalable Data Services and Solutions Dresden/Leipzig, German Centre for Integrative Biodiversity Research (iDiv), and Leipzig Research Center for Civilization Diseases, Universität Leipzig, Härtelstraße 16-18, 04107 Leipzig, Germany. ${ }^{4}$ Max Planck Institute for Math- ematics in the Sciences, Inselstraße 22, 04103 Leipzig, Germany. ${ }^{5}$ Fraunhofer Institute for Cell Therapy and Immunology, Perlickstraße 1, 04103 Leipzig, Ger- many. ${ }^{6}$ Department of Theoretical Chemistry, University of Vienna, Währinger Straße 17, 1090 Vienna, Austria. ${ }^{7}$ Center for non-coding RNA in Technology and Health, Grønegårdsvej 3, 1870 Frederiksberg C, Denmark. ${ }^{8}$ Santa Fe Institute, 1399 Hyde Park Rd., Santa Fe NM87501, USA.}

\section{Acknowledgements}

The authors want to thank David Langenberger for fruitful discussions on the subject of character loss, Petra Pregel and Jens Steuck for making work so much easier, and the Center for Information Services and High Performance Computing (ZIH) of the TU Dresden for allowing us to use their resources (see footnote 3). This work was funded by the Deutsche Forschungsgemeinschaft under the auspices of SPP-1174 Deep Metazoan Phylogeny (Project STA 850/2).

\section{Competing interests}

The authors declare that they have no competing interests.

\section{Availability}

The source code for gappy is available from https://github.com/alexdonath/ gappy. Machine readable files of simulated and real-life test data are available at http://www.bioinf.uni-leipzig.de/Publications/SUPPLEMENTS/17-002.
Consent for publication

Not applicable.

Ethics approval and consent to participate

Not applicable.

\section{Publisher's Note}

Springer Nature remains neutral with regard to jurisdictional claims in published maps and institutional affiliations.

Received: 3 June 2017 Accepted: 16 June 2018

Published online: 16 July 2018

References

1. Rivas E. Evolutionary models for insertions and deletions in a probabilistic modeling framework. BMC Bioinform. 2005;6:63.

2. Lèbre S, Michel CJ. A stochastic evolution model for residue insertion-deletion independent from substitution. Comput Biol Chem. 2010;34:259-67.

3. Redelings BD, Suchard MA. Incorporating indel information into phylogeny estimation for rapidly emerging pathogens. BMC Evol Biol. 2007;7:40.

4. Rivas E, Eddy SR. Probabilistic phylogenetic inference with insertions and deletions. PLoS Comput Biol. 2008;4:e1000172.

5. Dwivedi B, Gadagkar SR. Phylogenetic inference under varying proportions of indel-induced alignment gaps. BMC Evol Biol. 2009;9:211.

6. Luan PT, Ryder OA, Davis H, Zhang YP, Yu L. Incorporating indels as phylogenetic characters: impact for interfamilial relationships within Arctoidea (Mammalia: Carnivora). Mol Phylogenet Evol. 2013;66:748-56. https://doi. org/10.1016/j.ympev.2012.10.023.

7. Paško $Ł$, Ericson PG, Elzanowski A. Phylogenetic utility and evolution of indels: a study in neognathous birds. Mol Phylogenet Evol. 2011;61:76071. https://doi.org/10.1016/j.ympev.2011.07.021.

8. Nagy LG, Kocsubé S, Csanádi Z, Kovács GM, Petkovits T, Vágvölgyi C, Papp T. Re-mind the gap! Insertion-deletion data reveal neglected phylogenetic potential of the nuclear ribosomal internal transcribed spacer (ITS) of fungi. PLoS ONE. 2012;7:49794. https://doi.org/10.1371/journ al.pone.0049794.

9. Warnow T. Standard maximum likelihood analyses of alignments with gaps can be statistically inconsistent. PLoS Curr. 2012;4:1308.

10. Simmons MP. A confounding effect of missing data on character conflict in maximum likelihood and bayesian MCMC phylogenetic analyses. Mol Phylogenet Evol. 2014;80:267-80. https://doi.org/10.1016/j.ympev .2014.08.021.

11. Simmons MP, Ochoterena H. Gaps as characters in sequence-based phylogenetic analyses. Syst Biol. 2000;49:369-81.

12. Müller K. Incorporating information from length-mutational events into phylogenetic analysis. Mol Phylogenet Evol. 2006;38:667-76.

13. Teeling EC, Springer MS, Madsen O, Bates P, O'brien SJ, Murphy WJ. A molecular phylogeny for bats illuminates biogeography and the fossil record. Science. 2005;307:580-4.

14. Lloyd DG, Calder VL. Multi-residue gaps, a class of molecular characters with exceptional reliability for phylogenetic analyses. J Evol Biol. 1991;4:9-21.

15. Belinky F, Cohen O, Huchon D. Large-scale parsimony analysis of metazoan indels in protein-coding genes. Mol Biol Evol. 2010;27:441-51.

16. Ashkenazy H, Cohen O, Pupko T, Huchon D. Indel reliability in indel-based phylogenetic inference. Genome Biol Evol. 2014;6:3199-209. https://doi. org/10.1093/gbe/evu252.

17. Snir S, Pachter L. Tracing the most parsimonious indel history. J Comput Biol. 2011;18:967-86. https://doi.org/10.1089/cmb.2010.0325.

18. Blanchette M, Kent WJ, Riemer C, Elnitski L, Smit AF, Roskin KM, Baertsch R, Rosenbloom K, Clawson H, Green ED, Haussler D, Miller W. Aligning multiple genomic sequences with the threaded blockset aligner. Genome Res. 2004;14:708-15.

19. Paten B, Earl D, Nguyen N, Diekhans M, Zerbino D, Haussler D. Cactus: algorithms for genome multiple sequence alignment. Genome Res. 2011;21:1512-28. https://doi.org/10.1101/gr.123356.111. 
20. Boussau B, Daubin V. Genomes as documents of evolutionary history. Trends Ecol Evol. 2010;25:224-32.

21. Gu X, Li WH. The size distribution of insertions and deletions in human and rodent pseudogenes suggests the logarithmic gap penalty for sequence alignment. J Mol Evol. 1995:40:464-73.

22. Britten RJ, Rowen L, Williams J, Cameron RA. Majority of divergence between closely related DNA samples is due to indels. Proc Natl Acad Sci USA. 2003;100:4661-5.

23. Ogurtsov AY, Sunyaev S, Kondrashov AS. Indel-based evolutionary distance and mouse-human divergence. Genome Res. 2004;14:1610-6.

24. Lunter $\mathrm{G}$. Probabilistic whole-genome alignments reveal high indel rates in the human and mouse genomes. Bioinformatics. 2007;23:289-96.

25. Zhang Z, Gerstein M. Patterns of nucleotide substitution, insertion and deletion in the human genome inferred from pseudogenes. Nucleic Acids Res. 2003;31:5338-48.

26. Arndt PF, Hwa T. Regional and time-resolved mutation patterns of the human genome. Bioinformatics. 2004;20:1482-5.

27. Felsenstein J. Evolutionary trees from DNA sequences: a maximum likelihood approach. J Mol Evol. 1981;17:368-76.

28. Cartwright RA. DNA assembly with gaps (Dawg): simulating sequence evolution. Bioinformatics. 2005;21:31-8. https://doi.org/10.1093/bioin formatics/bti1200.

29. Fletcher W, Yang Z. INDELible: a flexible simulator of biological sequence evolution. Mol Biol Evol. 2009;26:1879-88.

30. Siepel A, Bejerano G, Pedersen JS, Hinrichs AS, Hou M, Rosenbloom K, Clawson H, Spieth J, Hillier LW, Richards S, Weinstock GM, Wilson RK, Gibbs RA, Kent WJ, Miller W, Haussler D. Evolutionarily conserved elements in vertebrate, insect, worm, and yeast genomes. Genome Res. 2005;15:1034-50.

31. ENCODE Consortium. Identification and analysis of functional elements in $1 \%$ of the human genome by the ENCODE pilot project. Nature. 2007:447:799-816.

32. Bauer DC, Bailey TL. Studying the functional conservation of cisregulatory modules and their transcriptional output. BMC Bioinform. 2008:9:220.

33. Larkin MA, Blackshields G, Brown NP, Chenna R, McGettigan PA, McWilliam H, Valentin F, Wallace IM, Wilm A, Lopez R, Thompson JD, Gibson TJ, Higgins DG. Clustal W and Clustal X version 2.0. Bioinformatics. 2007;23:2947-8

34. Edgar RC. MUSCLE: multiple sequence alignment with high accuracy and high throughput. Nucleic Acids Res. 2004;32:1792-7.

35. Notredame C, Higgins DG, Heringa J. T-Coffee: a novel method for fast and accurate multiple sequence alignment. J Mol Biol. 2000;302:205-17.

36. Löytynoja A, Goldman N. webPRANK: a phylogeny-aware multiple sequence aligner with interactive alignment browser. BMC Bioinform. 2010:11:579.

37. Subramanian AR, Kaufmann M, Morgenstern B. DIALIGN-TX: greedy and progressive approaches for segment-based multiple sequence alignment. Algorithms Mol Biol. 2008;3:6.

38. Katoh K, Kuma K, Toh H, Miyata T. MAFFT version 5: improvement in accuracy of multiple sequence alignment. Nucleic Acids Res. 2005;33:511-8.

39. Wang AX, Ruzzo WL, Tompa M. How accurately is ncRNA aligned within whole-genome multiple alignments? BMC Bioinform. 2007;8:417.

40. Do CB, Mahabhashyam MS, Brudno M, Batzoglou S. ProbCons: probabilistic consistency-based multiple sequence alignment. Genome Res. 2005;15:330-40

41. Gardner PP, Wilm A, Washietl S. A benchmark of multiple sequence alignment programs upon structural RNAs. Nucleic Acids Res. 2005;33:2433-9.

42. Lanfear R, Frandsen PB, Wright AM, Senfeld T, Calcott B. PartitionFinder 2: new methods for selecting partitioned models of evolution for molecular and morphological phylogenetic analyses. Mol Biol Evol. 2017;34:772-3. https://doi.org/10.1093/molbev/msw260.

43. Stamatakis A. RAxML version 8: a tool for phylogenetic analysis and postanalysis of large phylogenies. Bioinformatics. 2014;30:1312-3. https://doi. org/10.1093/bioinformatics/btu033.

44. Robinson DF, Foulds LR. Comparison of phylogenetic trees. Math Biosci. 1981:53:131-47

45. Penny $D$, Foulds LR, Hendy MD. Testing the theory of evolution by comparing phylogenetic trees constructed from five different protein sequences. Nature. 1982;297:197-200.
46. Estabrook GF, McMorris FR, Meacham CA. Comparison of undirected phylogenetic trees based on subtrees of four evolutionary units. Syst Biol. 1985:34:193-200.

47. Than C, Ruths D, Nakhleh L. PhyloNet: a software package for analyzing and reconstructing reticulate evolutionary relationships. BMC Bioinform. 2008;9:322.

48. Sand A, Holt MK, Johansen J, Brodal GS, Mailund T, Pedersen CNS. tqDist: a library for computing the quartet and triplet distances between binary or general trees. Bioinformatics. 2014;30:2079-80. https://doi.org/10.1093/ bioinformatics/btu157.

49. Stanhope MJ, Waddell VG, Madsen O, de Jong W, Hedges SB, Cleven GC, Kao D, Springer MS. Molecular evidence for multiple origins of Insectivora and for a new order of endemic African insectivore mammals. Proc Natl Acad Sci USA. 1998;95:9967-72.

50. Arnason U, Adegoke JA, Gullberg A, Harley EH, Janke A, Kullberg M. Mitogenomic relationships of placental mammals and molecular estimates of their divergences. Gene. 2008;421:37-51.

51. Murphy WJ, Pevzner PA, O'Brien SJ. Mammalian phylogenomics comes of age. Trends Genet. 2004;20:631-9.

52. Springer MS, Stanhope MJ, Madsen O, de Jong WW. Molecules consolidate the placental mammal tree. Trends Ecol Evol. 2004;19:430-8.

53. Murphy WJ, Pringle TH, Crider TA, Springer MS, Miller W. Using genomic data to unravel the root of the placental mammal phylogeny. Genome Res. 2007:17:413-21.

54. Nikolaev S, Montoya-Burgos Jl, Margulies EH, Rougemont J, Nyffeler B, Antonarakis SE. Early history of mammals is elucidated with the ENCODE multiple species sequencing data. PLoS Genet. 2007:3:2.

55. Prasad AB, Allard MW, Green ED. Confirming the phylogeny of mammals by use of large comparative sequence data sets. Mol Biol Evol. 2008;25:1795-808.

56. Kriegs JO, Churakov G, Kiefmann M, Jordan U, Brosius J, Schmitz J. Retroposed elements as archives for the evolutionary history of placental mammals. PLoS Biol. 2006;4:91.

57. Wildman DE, Uddin M, Opazo JC, Liu G, Lefort V, Guindon S, Gascuel O, Grossman LI, Romero R, Goodman M. Genomics, biogeography, and the diversification of placental mammals. Proc Natl Acad Sci USA. 2007;104:14395-400.

58. Simmons NB, Geisler JH. Phylogenetic relationships of Icaronycteris, Archeonycteris, Hassianycteris, and Palaeochiropteryx to extant bat lineages, with comments on the evolution of echolocation and foraging strategies in microchiroptera. Bull Am Mus Nat Hist. 1998;235:1-182.

59. Hutcheon JM, Kirsch JA, Pettigrew JD. Base-compositional biases and the bat problem. III. The questions of microchiropteran monophyly. Philos Trans R Soc Lond B Biol Sci. 1998;353:607-17.

60. Teeling EC, Madsen O, Van den Bussche RA, de Jong WW, Stanhope MJ, Springer MS. Microbat paraphyly and the convergent evolution of a key innovation in Old World rhinolophoid microbats. Proc Natl Acad Sci USA. 2002;99:1431-6.

61. Lin J, Chen G, Gu L, Shen Y, Zheng M, Zheng W, Hu X, Zhang X, Qiu Y, Liu $X$, Jiang C. Phylogenetic affinity of tree shrews to Glires is attributed to fast evolution rate. Mol Phylogenet Evol. 2014;71:193-200. https://doi. org/10.1016/j.ympev.2013.12.001.

62. Dessimoz C, Gil M. Phylogenetic assessment of alignments reveals neglected tree signal in gaps. Genome Biol. 2010;11:37.

63. Morrison DA, Ellis JT. Effects of nucleotide sequence alignment on phylogeny estimation: a case study of $18 \mathrm{~S}$ rDNAs of Apicomplexa. Mol Biol Evol. 1997;14:428-41.

64. Phillips A, Janies D, Wheeler W. Multiple sequence alignment in phylogenetic analysis. Mol Phylogenet Evol. 2000;16:317-30.

65. Wong K, Suchard M, Huelsenbeck J. Alignment uncertainty and genomic analysis. Science. 2008;319:473-6.

66. Blackburne BP, Whelan S. Class of multiple sequence alignment algorithm affects genomic analysis. Mol Biol Evol. 2013;30:642-53.

67. Hossain ASMM, Blackburne BP, Shah A, Whelan S. Evidence of statistical inconsistency of phylogenetic methods in the presence of multiple sequence alignment uncertainty. Genome Biol Evol. 2015;7:2102-16.

68. Capella-Gutiérrez S, Gabaldón T. Measuring guide-tree dependency of inferred gaps in progressive aligners. Bioinformatics. 2013;29:1011-7. https://doi.org/10.1093/bioinformatics/btt095.

69. Liu K, Linder CR, Warnow T. Multiple sequence alignment: a major challenge to large-scale phylogenetics. PLoS Curr. 2010;2:1198. 